\title{
ARTICLE
}

\section{Monte Carlo calculation of the neutron and gamma-ray distributions inside the LHD experimental building and shielding design for diagnostics}

\author{
Takeo Nishitani ${ }^{a^{*}}$, Kunihiro Ogawa ${ }^{\mathrm{a}, \mathrm{b}}$, Hiroki Kawase ${ }^{\mathrm{b}}$, Neng Pu ${ }^{\mathrm{b}}$, Tetsuo Ozaki ${ }^{\mathrm{a}}$ and Mitsutaka Isobe ${ }^{\mathrm{a}, \mathrm{b}}$ \\ ${ }^{a}$ National Institute for Fusion Science, National Institutes of Natural Sciences, 322-6 Oroshi, Toki, Gifu, 509-5292, Japan; \\ ${ }^{b}$ SOKENDAI (The Graduate University for Advanced Studies), 322-6 Oroshi, Toki, Gifu, 509-5292, Japan
}

\begin{abstract}
On the Large Helical Device (LHD), deuterium plasma experiments began in March 2017. In the plasma control, plasma heating and diagnostic systems, radiation sensitive components such as a programmable logic controller (PLC) and CCD sensors are commonly used. Three-dimensional distributions of neutron and gamma-ray in the LHD experimental building have been calculated by Monte Carlo code MCNP6 with the nuclear data library of ENDF B-VII.1 to prove the precise information on the radiation field and to introduce a countermeasure to the radiation. The total neutron flux in the torus hall of LHD is $\sim 10^{9} \mathrm{n} / \mathrm{cm}^{2} \bullet \mathrm{s}$ for the maximum neutron yield shot. The total neutron and gamma-ray fluxes in the basement are 1-2 order smaller than those in the torus hall, which is due to the streaming through penetrations in the floor concrete slab. The shielding design for the compact neutral particle analyzer (CNPA) is also carried out. It is found that $10 \%$ borated polyethylene thicker than $15 \mathrm{~cm}$ is necessary for the shield in all directions.
\end{abstract}

Keywords: Monte Carlo calculation; MCNP6; LHD; neutron; gamma-ray; shielding design; plasma diagnostics; compact neutral particle analyzer

\section{Introduction}

The Large Helical Device (LHD) [1] is one of the largest superconducting fusion machine, with a major radius of $R_{a x}=3.42-4.1 \mathrm{~m}$ and a maximum toroidal magnetic field of $B_{t}=3 \mathrm{~T}$. In March 2017, LHD started deuterium plasma experiments [2]. The licensed annual neutron budget is $2.1 \times 10^{19}$ neutrons/year. The maximum neutron production rate is expected to be 1.9 $\times 10^{16} \mathrm{n} / \mathrm{s}$ [2].

Highly integrated electronic components such as a programmable logic controller (PLC) and sensors such as a charge-coupled device (CCD) are indispensable in the control, plasma heating and diagnostic systems. Those components are considered to be very sensitive to neutrons and gamma-rays. Indeed, the neutron and gamma-ray irradiation tests on those components indicate the possibility of errors or damages even in the radiation field expected in LHD [3]. For the deuterium plasma experiments, relocation to the basement or the peripheral region of the torus hall, and shielding has been undertaken for control and diagnostic systems. Three-dimensional distributions of neutron and gamma-ray have been calculated by Monte Carlo code to prove the precise information on the radiation field and to introduce a countermeasure to the radiation.

Some diagnostic equipment has to remain in the torus

*Corresponding author. Email: nishitani.takeo@nifs.ac.jp hall, mainly because those have to face to the plasma directly. Radiation shielding is required for such diagnostics equipment to avoid radiation induced noises and errors. In this paper, shielding design for the compact neutral particle analyzer (CNPA) [4,5] is presented as one of the examples.

\section{Calculation code and model}

In this calculation, a General Monte Carlo N-Particle Transport Code version 6 (MCNP-6) [6] is used with the nuclear data library of ENDF/B-VII.1 [7].

The schematic view of the MCNP calculation model for LHD and the LHD experimental building is shown in Figure 1, which is drawn with SuperMC code [8,9]. In the previous work [10], $36^{\circ}$ sector model of LHD including $36^{\circ}$ sector cylindrical model of the concrete floor, wall and ceiling was used to estimate the radiation field arround the LHD machine, where the basement was not modeled. In this work, LHD and the LHD experimental building including the basement are modeled manually from the CAD drawings. Especially, penetrations in the floor concrete slab are carefully modeled, because the basement is the first candidate of the relocation place for electronic cubicles. In the torus hall, LHD itself and the interferometer support are modeled. The other apparatus such as neutral beam (NB) injectors and vacuum pumps are not included so far. NB injectors and vacuum pumps are not so large volume 
compared to LHD, however, may affect the radiation field in the torus hall. Therefore those components will be modeled in near future. MCNP could not treat helical surfaces, thus the helical coils and the case structures are divided by $6^{\circ}$ toroidal angle pitch, and those components are assumed to be toroidally symmetric in a toroidal pitch angle [10]. The torus hall basement is separated by a mezzanine stage ( $Z=-8 \mathrm{~m}$ from the floor level), made of stainless plates and frames to B1 and B2 levels, which is not modeled because the mass is not so large.

The helical and poloidal coils are modeled by homogenized materials, respectively, based on each composition such as superconductors of $\mathrm{NbTi}$, electric insulators, copper stabilizing conductors, and liquid helium. The material of the vacuum vessel and the support structure is stainless steel (SS) $316 \mathrm{~L}$, and that of the cryostat is SS304. The LHD experimental building is built with normal concrete.

The LHD plasma, which is a volumetric neutron source, is modeled by the circular torus, and is divided into five regions in minor radius whose neutron emission rates are determined to be a neutron source profile expected by a plasma simulation. The source neutron energy is assumed to be $99.5 \%$ of $2.45 \mathrm{MeV}$ and $0.5 \%$ of $14.1 \mathrm{MeV}$. The $14 \mathrm{MeV}$ neutron is generated by reaction between the bulk deuteron and the energetic triton from the $\mathrm{d}(\mathrm{d}, \mathrm{n}) \mathrm{t}$ reaction [11]. The source neutrons are assumed to be isotropic.

The calculations have been executed on a LINUX workstation with 36 cores of RC Viento 3600XB, Real Computing. Inc.
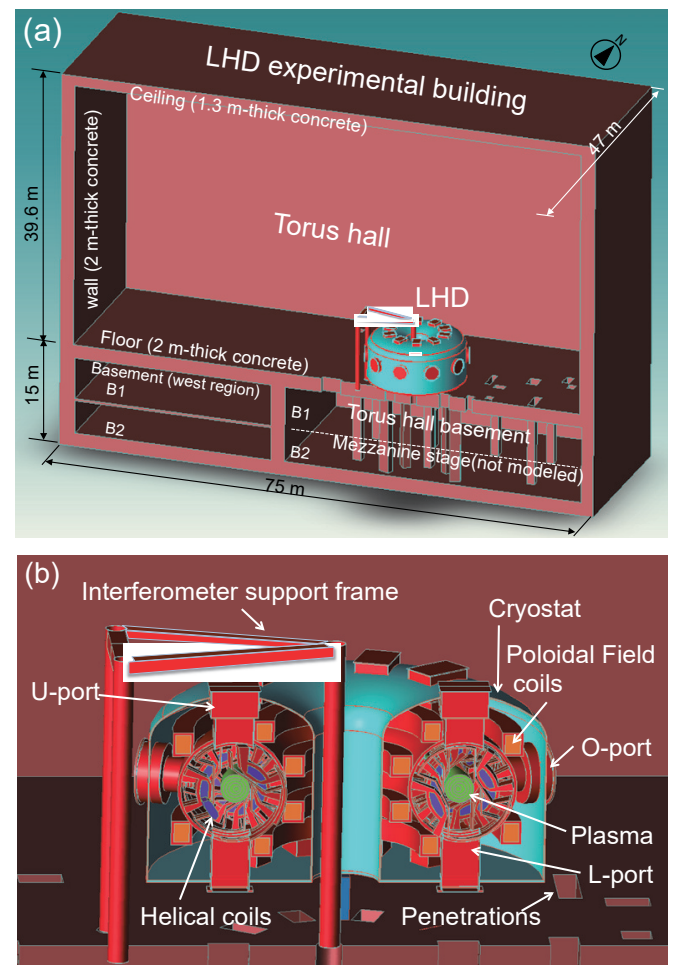

Figure 1. Schematic view of the MCNP calculation model for LHD and the experimental building. (a) overall view and (b) expanded cut view of LHD.

\section{Calculation results}

\subsection{Neutron flux distributions}

Three-dimensional neutron flux distributions have been calculated by using the mesh tally function of MCNP. Figure 2 shows the contour plots of the neutron flux distribution on (a) the vertical plane at the center of LHD, (b) the horizontal plane at the equatorial level $(\mathrm{Z}=$ $5.5 \mathrm{~m}$ from the floor level), and (c) the horizontal plane at $1 \mathrm{~m}$ above the mezzanine stage level in the torus hall basement.

(a)

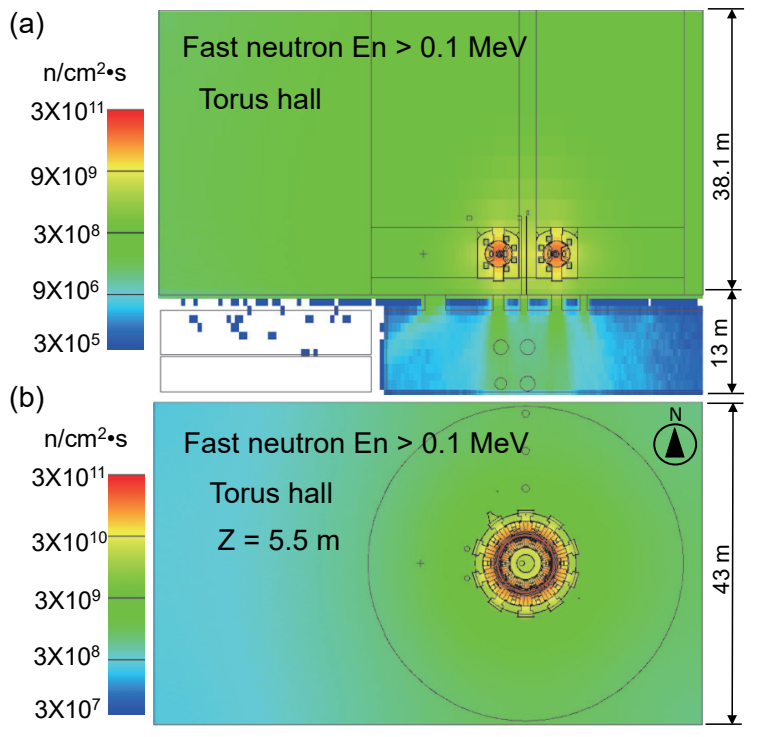

(c) $\mathrm{n} / \mathrm{cm}^{2} \cdot \mathrm{s}$

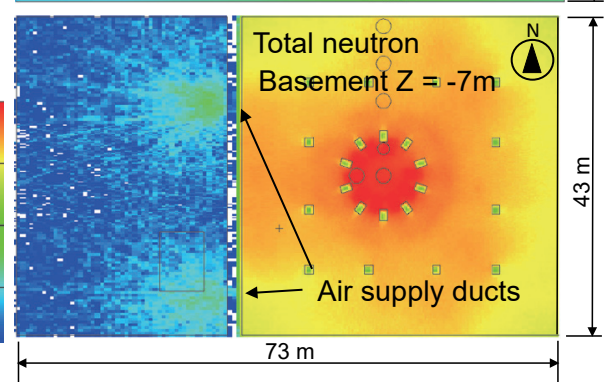

Figure 2. Contour plots of the neutron flux distribution at the neutron emission rate of $1.9 \times 10^{16} \mathrm{n} / \mathrm{s}$ on (a) the vertical plane at the center of LHD, (b) the horizontal plane at the equatorial level $(Z=5.5 \mathrm{~m})$, and (c) the horizontal plane $(\mathrm{Z}=$ $-7 \mathrm{~m})$ in the torus hall basement.

In the torus hall, neutron streaming from horizontal ports is not so clear, which is provably due to the thick port flange with $60 \mathrm{~mm}$ in thickness. Also there are not so large differences of the neutron flux in the direction of north, east and south from the center of LHD, which can be explained by the neutron reflection from the wall.

Neutron streaming from floor penetrations is clearly observed. In the torus hall basement, total neutron flux is rather high, typically $\sim 1 \times 10^{8} \mathrm{n} / \mathrm{cm}^{2} \bullet \mathrm{s}$, just under the LHD machine. That of other region is $\sim 10^{7} \mathrm{n} / \mathrm{cm}^{2} \bullet$ s. Those neutron fluxes in the torus hall basement are much larger than we expected. Neutron streaming through air supply ducts in the wall between the torus hall basement and the basement west region is identified. 
We have to consider the radiation effects on the equipment installed in the basement west region, where we expected that the neutron and gamma-ray flux would be very low.

Also three-dimensional gamma-ray flux distributions are calculated, which is similar to that of neutrons.

\subsection{Neutron and gamma-ray spectra}

Neutron and gamma-ray spectra in the LHD experimental building have been calculated by using a track length estimation tally, so-called cell tally. Those tally cells are spheres of $1 \mathrm{~m}$ diameter and $2 \mathrm{~m}$ diameter in the torus hall and the basement, respectively. Typical size of the electronics cubicle is $0.9 \times 0.9 \times 2 \mathrm{~m}^{3}$, therefore tally cells of spheres with $1 \mathrm{~m}$ diameter are adopted in the torus hall. In the basement, the diameter of tally cells is $2 \mathrm{~m}$ in order to obtain sufficient statistics.

(a)

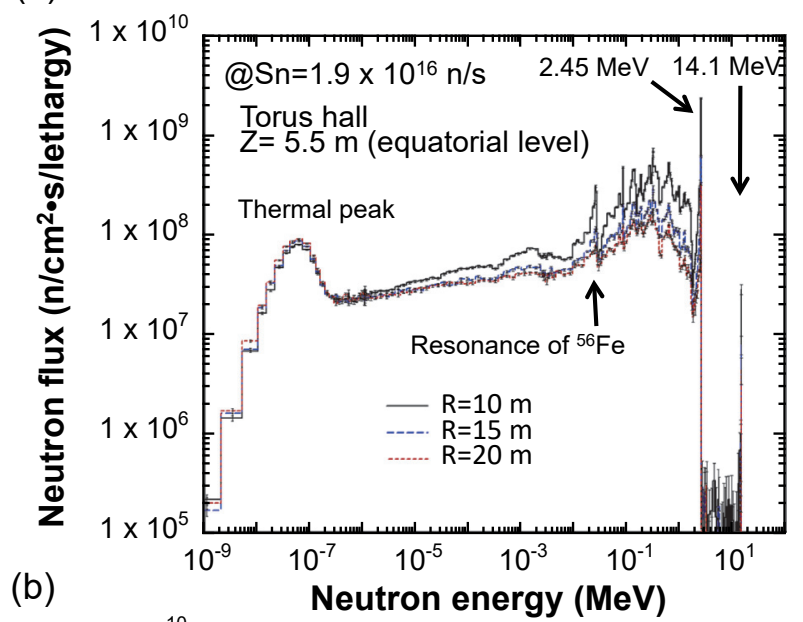

(b)

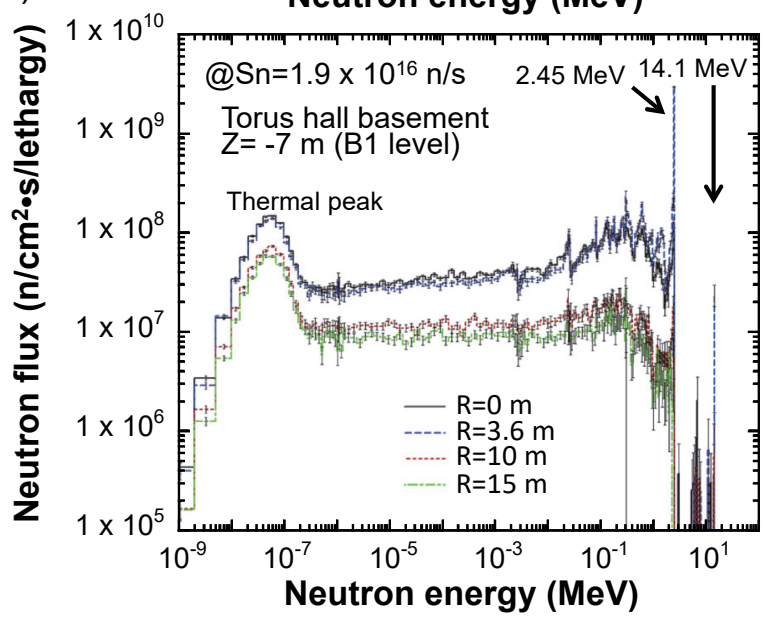

Figure 3. Neutron spectra at the neutron emission rate of 1.9 $\times 10^{16} \mathrm{n} / \mathrm{s}$ in (a) the torus hall on the equatorial level $(\mathrm{Z}=5.5$ $\mathrm{m})$, and (b) the torus hall basement on the B1 level $(\mathrm{Z}=-7 \mathrm{~m})$.

Figure 3 shows neutron spectra in the torus hall on the equatorial level, and the torus hall basement on the B1 level at the shot with the maximum neutron emission rate, where the number of energy bins is 199. Virgin neutrons of $2.45 \mathrm{MeV}$ and $14 \mathrm{MeV}$ are clearly seen not only in the torus hall but also in the torus hall basement, which indicates that those neutron in the torus hall basement come from the penetrations under the LHD machine. It can be considered that several peaks and gaps in $\mathrm{keV}$ region are corresponding to resonances of ${ }^{56} \mathrm{Fe}$ and other structural materials. The thermal neutron peak is formatted in all spectra. There is not so large difference of the neutron flux and spectrum among the major radius of 10,15 and $20 \mathrm{~m}$ in the torus hall. In the torus hall basement, neutron fluxes of the central region $(\mathrm{R}<4 \mathrm{~m})$ are about 1 order smaller than those of the peripheral region in the torus hall. On the other hand, neutron fluxes of the peripheral region are about 2 order smaller the those in the torus hall.

Also gamma-ray spectra in the torus hall and the torus hall basement are shown in Figure 4, where the number of energy bins is 80 . Prompt gamma-rays from concrete components, such as $\mathrm{H}(\mathrm{n}, \gamma),{ }^{56} \mathrm{Fe}\left(\mathrm{n}, \mathrm{n}\right.$ '), ${ }^{29} \mathrm{Si}(\mathrm{n}, \gamma)$, and ${ }^{56} \mathrm{Fe}(\mathrm{n}, \gamma)$ are identified. Spectrum shapes are almost same in the torus hall and the torus hall basement. Gamma-ray fluxes in the torus hall basement are 1-2 order smaller than those in the torus hall.

(a)

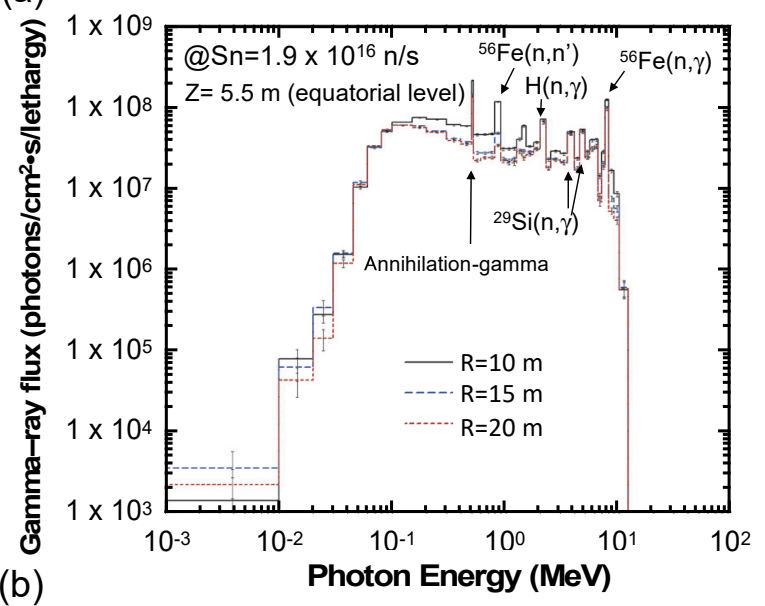

(b)

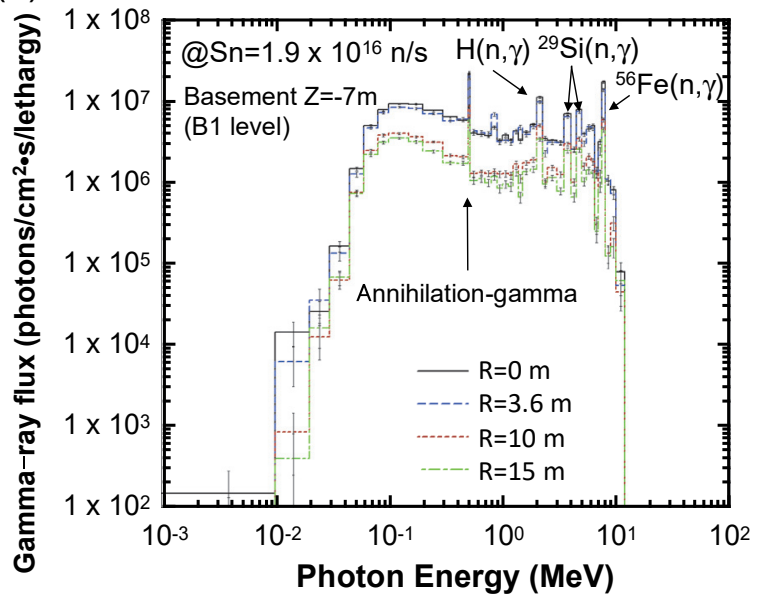

Figure 4. Gamma-ray spectra at the neutron emission rate of $1.9 \times 10^{16} \mathrm{n} / \mathrm{s}$ in (a) the torus hall on the equatorial level $(\mathrm{Z}=$ $5.5 \mathrm{~m})$, and (b) the torus hall basement on the B1 level ( $\mathrm{Z}=-7$ $\mathrm{m})$. 


\section{Example of shielding design for diagnostic apparatus}

The Compact Neutral Particle Analyzer (CNPA) is a E//B type neutral particle energy analyzer using permanent magnets as shown in Figure 5, which has been developed by the Ioffe Institute, Russia [5]. CNPA is installed on the diagnostic platform and connected to the outside horizontal port (O-port) via a flight tube.

The specification of CNPA indicates that the maximum allowed neutron flux at the NPA location is 1 $\times 10^{7} \mathrm{n} / \mathrm{cm}^{2} \bullet \mathrm{s}$ [5], where the most sensitive component is the detector array. To meet this request, the neutron shield is designed within the weight limit of the diagnostic stage. Neutron fluxes are calculated for several cases with different shielding material (polyethylene or $10 \%$ borated polyethylene) and the thickness. Finally, $15 \mathrm{~cm}$ thick borated polyethylene (front side: $15+10 \mathrm{~cm}$ ) is adopted as shown in Figure 5, where neutron flux inside CNPA is estimated to be 0.71 $\times 10^{7} \mathrm{n} / \mathrm{cm}^{2} \bullet \mathrm{s}$. The overall size of the shield is $1.05 \mathrm{~m}^{\mathrm{w}} \times$ $0.5 \mathrm{~m}^{\mathrm{d}} \times 0.85 \mathrm{~m}^{\mathrm{h}}$.

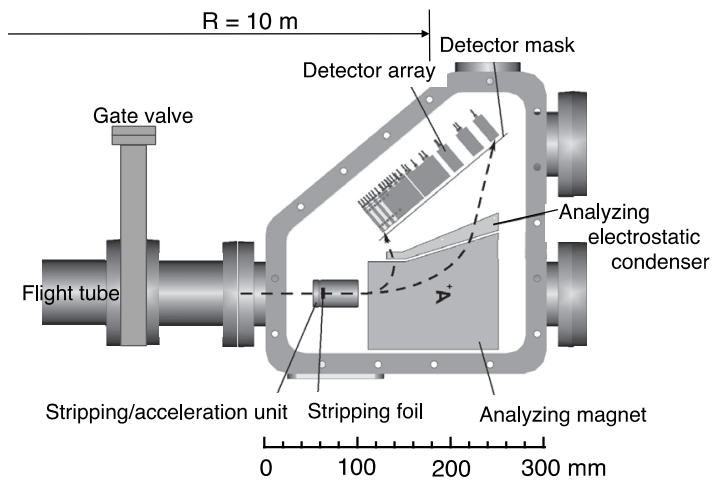

Figure 4. Schematic view of the Compact Neutral Particle Analyzer (CNPA) on LHD.

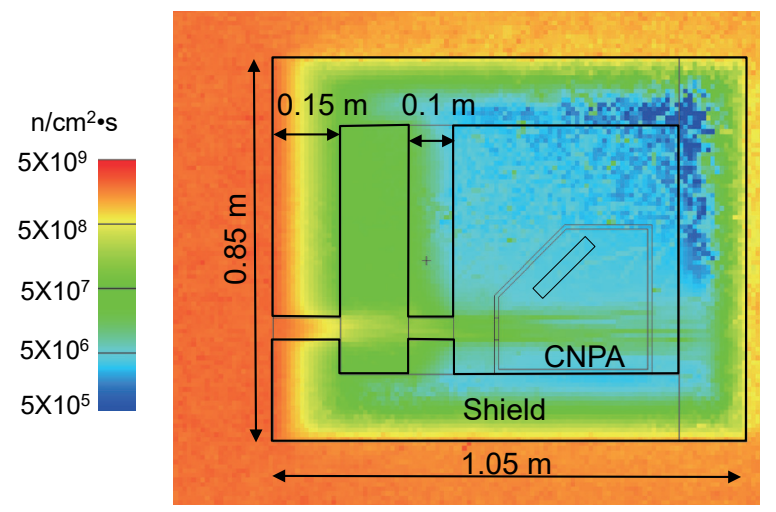

Figure 5. Neutron flux distribution around CNPA with the shield of $15 \mathrm{~cm}$ thick borated polyethylene (front side: $15+10$ $\mathrm{cm})$.

\section{Summary}

Three-dimensional distributions of neutron and gamma-ray in the LHD experimental building have been calculated by MCNP6 with the nuclear data library of
ENDF B-VII.1 to prove the precise information on the radiation field for the shielding and/or the relocation of diagnostic and control equipment. The total neutron and gamma-ray fluxes in the basement are $10^{-2}-10^{-1}$ of those in the torus hall, which is larger than we expected previously. The shielding design for CNPA is also carried out. The shield of $15 \mathrm{~cm}$ thick borated polyethylene (front side: $15+10 \mathrm{~cm}$ ) is adopted.

\section{Acknowledgements}

This work is supported partly by the LHD project budget (ULGG801). One of the authors, T.N., wishes to thank Drs. M. Ishikawa and T. Shimada for their support of the SuperMC usage.

\section{References}

[1] O. Kaneko, et al., Extension of operation regimes and investigation of three-dimensional currentless plasmas in the Large Helical Device, Nuclear. Fusion 53 (2014), p. 104015.

[2] M. Osakabe and LHD Experimental Group, Preparation and commissioning for the LHD deuterium experiment, submitted to IEEE Trans. Plasma Science.

[3] K. Ogawa, et al., Investigation of irradiation effects on highly integrated leading edge electronic components of diagnostics and control systems for the LHD deuterium operation, Nuclear Fusion 57 (2017), p. 086012.

[4] T. Ozaki, et al., Pellet charge exchange helium measurement using neutral particle analyzer in large helical device, Rev. Sci. Instrum. 79 (2008), p.10E518.

[5] Neutral particle analyzer, Ioffe institute, http://www.ioffe.ru/ACPL/npd/npa00.htm.

[6] D.B. Pelowits (Ed.), MCNP6 Users Manual, LA-CP-13-00634, Los Alamos National Laboratory, (2013).

[7] M.B. Chadwick, et al., ENDF/B-VII.1 Nuclear data for science and technology: Cross sections, covariances, fission product yields and decay data, Nuclear Data Sheets 112 (2011), pp. 2887-2996.

[8] Y. Wu and FDS Team, Benchmarking of CAD-based SuperMC with ITER benchmark model, Fusion Eng. and Des. 84 (2009), pp. 1987-1992.

[9] Y. Wu, et al., CAD-based Monte Carlo program for integrated simulation of nuclear system SuperMC, Ann. Nucl. Energy 82 (2015), pp. 161-168.

[10]T. Nishitani, K. Ogawa and M. Isobe, Radiation field estimation for the diagnostic and control components by Monte Carlo neutronics calculations with LHD 3-dimensional modeling, Plasma and Fusion Research 11 (2016), p. 2405057.

[11]M. Isobe, et al., Neutron diagnostics in the Large Helical device, submitted to IEEE Trans. Plasma Science. 